\title{
Reflets
}

Revue ontaroise d'intervention sociale et communautaire

\section{Le mouvement des communautés en santé dans les comtés unis de Prescott-Russell}

\section{Jean-Marc Dupont et Johanne Nolin}

Volume 1, numéro 2, automne 1995

La santé communautaire en Ontario français : défis et espoirs

URI : https://id.erudit.org/iderudit/026094ar

DOI : https://doi.org/10.7202/026094ar

Aller au sommaire du numéro

Éditeur(s)

Reflets : Revue ontaroise d'intervention sociale et communautaire

ISSN

1203-4576 (imprimé)

1712-8498 (numérique)

Découvrir la revue

Citer cet article

Dupont, J.-M. \& Nolin, J. (1995). Le mouvement des communautés en santé dans les comtés unis de Prescott-Russell. Reflets, 1(2), 251-260.

https://doi.org/10.7202/026094ar

Tous droits réservés (C) Reflets : Revue ontaroise d'intervention sociale et communautaire, 1995
Ce document est protégé par la loi sur le droit d'auteur. L'utilisation des services d'Érudit (y compris la reproduction) est assujettie à sa politique d'utilisation que vous pouvez consulter en ligne.

https://apropos.erudit.org/fr/usagers/politique-dutilisation/ 


\section{Le mouvement des communautés en santé dans les comtés unis de Prescott-Russell}

\section{Jean- $M$ arc $D$ upont}

Expert-conseil en santé communautaire et animateur communautaire

C oalition des communautés en santé de l' 0 ntario

\section{Johanne $\mathbf{N}$ olin}

A gente de développement communautaire, P rescott- $R$ ussell en santé

O $\mathrm{n}$ a beaucoup écrit sur le mouvement des $\mathrm{C}$ ommunautés en santé (cs) au cours des dix dernières années. En présentant l'approche des cs aux lecteurs de R eflets (groupes communautaires intéressés, aux politiciens municipaux et aux citoyens), nous sommes convaincus de deux choses: premièrement, bien que traitant de sujets similaires, toutes les communautés sont différentes; et deuxièmement, la question $\ll Q$ uelle est la définition d'une communauté en santé?» est presque inévitable. La réponse est pourtant très simple: «U ne communauté en santé est ce que vous désirez en faire!». Bien que le mouvement des $C$ ommunautés en santé soit une approche de développement communautaire reposant sur des valeurs précises comme la santé et la qualité de vie dans la communauté, la meilleure façon de le décrire est d'examiner les projets innovateurs et axés vers l'action qui en découlent. En fait, plutôt que d'encourager les communautés à faire autre chose, le mouvement les encourage à faire les mêmes choses, mais autrement. Comme nous le verrons dans le présent article, la force et la viabilité des projets reposent sur l'importance accordée aux partenariats multisectoriels, à la grande participation de la communauté et à l'appui du rôle et de la responsabilité des 
municipalités à titre d'agents essentiels de promotion de la santé. Q u'il soit perçu comme une approche ou comme la base de projets concrets, le mouvement des Communautés en santé de Prescott- $R$ ussell bat son plein et des choses positives se produisent.

\section{Qu'est ce qu'une communauté en santé?}

\section{Une vision}

Lorsqu'on pose cette question, les gens pensent à beaucoup de choses: de l'air et de l'eau propres, des rues sans danger, des logements adéquats à prix raisonnable, des emplois satisfaisants qui fournissent un revenu convenable, l'accessi bilité à des espacesverts, un sentiment d'appartenance, etc. En fait, l'environnement social et physique dans lequel nous vivons, travaillons et nous détendons a une bien plus grande influence sur notre santé et notre qualité de vie que n'importe quelle intervention du système médical.

\section{Une nouvelle compréhension de ce que sont les déterminants de la santé}

Au fil des ans, nous avons compris que les facteurs déterminants de la santé des gens et de la qualité de vie des communautés vont beaucoup plus loin que la simple accessibilité aux services de santé. C es déterminants, y compris le revenu, l'emploi, l'éducation, la sécurité, le logement, la nourriture, la stabilité de l'écosystème et la viabilité des ressources sont aujourd' hui indissociables de la santé et du bien-être d'une communauté et de ses membres. 


\section{Un rôle important pour la municipalité}

Les gouvernements municipaux jouent un rôle important dans plusieurs domaines qui touchent la santé d'une communauté: la planification urbaine et la conception des quartiers, des parcs, des routes, des systèmes de transport en commun, des installations récréatives et des stratégies de développement économique, la propreté de l'eau, les égouts et la cueillette des déchets, les règlements contre le tabagisme, l'épanouissement social, etc.

«U ne communauté en santé crée et améliore constamment l'environnement physique et sodal et favorise I'expansion des ressources communautaires qui permettent aux gens de $s^{\prime}$ entraider pour mieux vivre et développer au maximum leur potentiel.» (Traduction libre, $\mathrm{H}$ ancock et $\mathrm{D}$ uhl, 1986)

\section{Quelques concepts de base}

L'approche des Communautés en santé a été adoptée par des villes du Canada et de l'A ustralie, de l'A frique et du Japon. Bien que les initiatives des nombreuses villes adhérentes diffèrent, les deux concepts suivants sont à la base même des discussions tenues partout dansle monde au sujet des C ommunautés en santé: premièrement, les déterminants de la santé et de la qual ité de vie touchent à plusieurs secteurs, deuxièmement, en raison de l'influence qu'elles exercent dans un grand nombre de ces secteurs et de la proximité qu'elles ont avec leurs citoyens, les municipalités ont un rôle très important à jouer dans la santé de leur communauté.

L'approche des Communautés en santé repose sur quatre stratégies:

- une grande participation des gens de toutes les conditions sociales en vue d'améliorer la santé de leur communauté; 
- une participation intersectorielle, donc des représentants de nombreux secteurs de la communauté (citoyens, entreprises, églises, fournisseurs de services, planificateurs, groupes environnementaux) se forgeant une vision d'une communauté en santé et cherchant dans leur travail de tousles jours des façons d'atteindre les buts visés;

- un gouvernement local qui s'engage à ce que chacun des services sous sa juridiction (comme les parcs, les services de loisirs, les travaux publics) ait la même vision d'une communauté en santé et se donne les moyens pour appuyer cette vision;

- et finalement, une politique publique visant la santé, puisque l'amélioration de la santé d'une communauté ne repose pas toujours sur l'offre d'un plus grand nombre de services, mais sur l'examen de façons de promouvoir la santé au sens large grâce, par exemple, à un changement de législation.

Ici en 0 ntario, comme ailleurs dans le monde, le principe des Communautés en santé repose aussi sur le fait que les communautés doivent faire face aux situations engendrées par les conditions économiques, environnementales et sociales actuelles. Les gouvernements municipaux, les groupes communautaires, les organismes et les résidents doivent donc s'unir pour tenter de résoudre les questions qui les préoccupent directement. $M$ alheureusement, le financement offert par les gouvernements fédéral et provinciaux n'est généralement pas distribué de façon à promouvoir la collaboration intersectorielle et ne fournit pas aux communautés l'aide dont elles ont besoin pour réunir les différents membres autour de la même table dans le cadre d'une action concertée. De ce fait, les communautés ne peuvent pas profiter pleinement du soutien disponible et gaspillent du temps, de l'énergie et de l'argent.

Le but de l'approche des C ommunautés en santé est d'aider les membres de la communauté à cerner les facteurs économiques, environnementaux et sociaux qui affectent leur qualité de vie et de travailler avec leur gouvernement municipal pour aborder ces questions en utilisant au maximum les ressources locales. 
Figure 1

\section{Une communauté en santé}

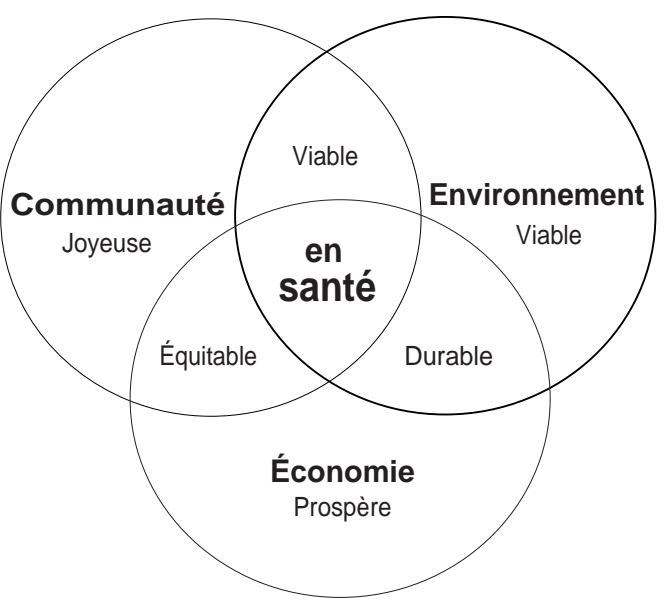

U ne communauté en santé, en somme, c'est un projet de promotion de la santé et de la qualité de vie, une stratégie de concertation entre les citoyens et les décideursmunicipaux. C ette stratégie permet aux citoyens de participer activement au développement du mieux-être collectif et d'influencer positivement les décisions qui touchent leur qualité de vie. Q uant aux décideurs, elle leur permet de jouer leur rôle de leader communautaire tout en les encourageant à considérer les facteurs santé et qualité de vie dans l'ensemble de leurs actions.

II est important de reconnaître que l'expansion de cet intéressant réseau régional va de pair avec la croissance de la C oalition des communautés en santé de l'O ntario (ccso) au niveau provincial, et que la ccso fait à son tour partie d'un mouvement international sans cesse croissant de communautés en santé.

La ccso est un réseau de coalitions locales réunissant des groupes communautaires et des organismes provinciaux qui partagent et appuient une même vision des communautés en santé. Les membres de la C oalition apportent une gamme de compétences professionelles et organisationnelles aux niveaux provincial et local. 
La C oalition est financée par le ministère de la Santé de l'O ntario et les fonctions administratives qui s'y rattachent sont assumées par le bureau provincial des C ommunautés en santé de l'O ntario situé àToronto. Bien que ce bureau compte peu de personnel sur place, une intervention directe décentralisée est fournie par des employés et des personnes intéressées aux communautés en santé dans les différentes régions de la province.

\section{Le concept Communautés en santé de Prescott-Russell}

«C e ne sont pas les médecins ou les hôpitaux qui contribuent le plus à la santé d'une nation mais plutôt les gouvernements locaux.» ( $D^{r}$ Jessie Parfitt)

Tout comme au bureau provincial, la dynamique de l'approche C ommunautés en santé de Prescott- $R$ ussell repose sur une coopération multisectorielle, flexible, faisant place à une participation réelle des citoyens. Cette coopération, qui vise un changement à long terme, s'exerce dans des actions concrètes, non conflictuelles et réalisables à court terme à partir des ressources existantes.

C ontrairement à d'autres régions, et grâce au partenariat et à la vision du Bureau de santé de l'est de l'O ntario et du C onseil de planification de Prescott- $R$ ussell, ces comtés profitent non seulement d'un animateur communautaire de la Coalition de I'O ntario, mais de deux employés additionnels engagés par les organisations susmentionnées. Ces derniers ont pour tâche de veiller à ce que toutes les municipalités des comtés unis deviennent des communautés en santé! L'engagement du Bureau de santé de l'est de l'O ntario et du Conseil de planification de Prescott- $R$ ussell est sans contredit un modèle pour le reste de la province. 
Le concept est fondé sur la participation communautaire et municipale. Les conseils municipaux sont invités à manifester leur appui en adoptant une résolution municipale appropriée. Les problèmes de la communauté ne deviennent toutefois pas les problèmes du conseil. Ils demeurent nos problèmes. Dans un tel contexte, la dynamique change complètement: nous cessons de nous lancer la balle, un partage s'installe, un partenariat solide prend racine. II n'y a pas de secret. La clé du succès repose tout simplement sur la collaboration des partenaires et sur la participation accrue des citoyens.

Toutes les façons d'implanter l'approche Communautés en santé dans les municipalités sont bonnes. II faut par contre prendre le temps de bien connaître la communauté dans laquelle nous devons travailler. C eci peut supposer de nombreuses rencontres avant de trouver le groupe ou la personne qui va faire avancer les choses. II faut surtout croire en l'approche, en parler partout et profiter de toutes les occasions pour présenter le concept au gens.

Voici quelques exemples d'initiatives amorcées dans diverses municipalités des comtés de Prescott- $R$ ussell. Le $C$ anton de Clarence, comme bien d'autres municipalités, fait face à de sérieuses compressions budgétaires. Les besoins des citoyens demeurent toutefois les mêmes. Q ue faire? Le conseil municipal a décidé de passer une résolution exprimant sa participation àl'approche des Communautés en santé. U n élu municipal a pris le dossier en main afin de donner la chance à sa communauté d'exprimer ses besoins. La participation des citoyens, d'organismes et de groupes sociaux a grandement aidé le comité C larence en santé à élaborer son plan d'action et à cerner les priorités de la communauté.

Embrun et R ussell, deux municipalités bien différentes, I'une francophone et l'autre anglophone, partagent un projet en commun : développer l'approche C ommunautés en santé. Le projet a commencé grâce à la participation des chambre de commerce et du conseil municipal. $R$ éunir les ressources, les connaissances et l'expérience des deux communautés de langue et de culture différentes sont les principaux objectifs visés par E mbrun- $R$ ussell en 
santé. Au dire d'un intervenant, la situation économique actuelle nous fait voir la santé d'une façon bien différente et nous fait travailler avec de nouveaux partenaires, comme la chambre de commerce, le secteur privé, le centre d'alphabétisation, le centre d'emploi, etc.

$\mathrm{R}$ odkland $\mathrm{C}$ ommunauté en santé travaille présentement en collaboration avec l'équipe du plan directeur. Pour être à l'écoute des citoyens, Communauté en santé a organisé plusieurs réunions, y compris une session de visionnement de film. Le conseil municipal appuie les démarches, puisque c'est une occasion idéale d'entendre les commentaires des citoyens de façon constructive! U n conseiller assure la représentation du Comité en santé à la table du conseil.

L'approche Communautés en santé de Prescott-R ussell évolue rapidement. Elle donne une nouvelle vitalité aux comtés unis, lesquels supportent d'ailleurs l'approche et incitent les municipalités à promouvoir le concept chez elles. Bon nombre de municipalités ont maintenant passé une résolution municipale les engageant à la promouvoir. Tout en travaillant en concertation avec le bureau provincial des C ommunautés en santé de l'O ntario pour assurer le lien entre lescommunautés et le mouvement provincial, le bureau autonome de Prescott- $R$ ussell s'engage auprès des municipalités à fournir l'aide nécessaire pour démarrer les initiatives qui les aideront à se joindre au nombre grandissant de communautés en santé.

\section{Les défis à venir}

$\mathrm{N}$ ous avons encore beaucoup à apprendre sur les $\mathrm{C}$ ommunautés en santé: comment établir la structure des comités de façon à mieux répondre aux conditions de chacun; comment appuyer des initiatives orientées vers l'action; comment amener les conseilsmunicipaux à considérer les $C$ ommunautés en santé comme une approche pouvant les aider à relever les défis créés par les 
anciennes méthodes de travail; et comment promouvoir, du point vue administratif, le mouvement des C ommunautés en santé aux niveaux municipal, régional, provincial et international. La vraie réussite de l'approche sera toujoursévaluée en fonction de ce qui se fait au niveau local. Comme l'indique clairement la réussite de Prescott- $R$ ussell, les gouvernements municipaux peuvent accepter une responsabilité de promoteurs de la santé. Les différents secteurs d'une communauté peuvent travailler ensemble sans protéger leur territoire, et les citoyens peuvent avoir leur mot à dire lorsqu'il s'agit d'établir des priorités. Les actions coopératives améliorent sans contredit la qualité de vie des communautés. C'est très simple... Tout le monde bénéficie de l'approche des C ommunautés en santé!

\section{Comment s'y prendre pour devenir une communauté en santé?}

Pour qu'une communauté soit reconnue comme une communauté en santé et devienne membre de la Coalition provinciale, elle doit adopter, par l'entremise de son conseil de ville, une résolution comprenant une déclaration de principe qui souscrit à la philosophie véhiculée par le concept de C ommunautésen santé. D ans cette déclaration, la municipalité s'engage devant ses citoyens et citoyennesà devenir une municipalité en santé en agis sant à la fois comme animatrice et comme participante du projet.

Un engagement doit aussi être pris en vue de la formation d'un comité multisectoriel comprenant des représentants de la municipalité et d'au moins un organisme d'un autre secteur. $\mathrm{Ce}$ comité devrait avoir pour mandat de développer et proposer, et éventuellement réaliser, un ou des projets visant à améliorer la santé ou la qualité de la vie dans la communauté.

La municipalité doit de plus s'engager, en principe, à affecter un minimum de ressources humaines, matérielles ou financières 
à la mise en oeuvre des projets retenus par le conseil municipal. II s'agit d'ailleurs davantage d'une réorientation des ressources existantes que de nouveaux investissements. U n modèle de résolution d'adhésion est disponible au bureau provincial ou au bureau de Prescott- $R$ ussell.

Les collectivités ou les groupes communautaires organisés qui appuient le concept des communautés en santé et qui se sont engagés à obtenir le soutien du conseil municipal peuvent adhérer à Prescott- $R$ ussell en santé ainsi qu'à la C oalition provinciale. 